\title{
Сравнение регрессионных моделей получения параметров уравнения изотермы адсорбции Ленгмюра методом компьютерного моделирования
}

\author{
Нгуен Фук Као, Везенцев А.И., Данг Минь Тхуи, Перистая Л.Ф. \\ Белгородский государственный нацииональный исследовательский университет (НИУ“БелГУ”), \\ Белгород
}

Поступила в редакцию 1.03.2018 г.

DOI: https://doi.org/10.17308/sorpchrom.2018.18/597

В работе представлены результаты сравнения оценки параметров, полученных методом наименьших квадратов из двух традиционных линейных и одной нелинейной форм уравнения Ленгмюра на основе исследования изотермы адсорбции ионов $\mathrm{Pb}^{2+}$ на монтмориллонитовых глинах месторождений (Поляна, Нелидовка - Белгородская область, Россия и Там Бо провинция Лам Донг, Вьетнам). Методом симуляции Монте-Карло построены $10^{4}$ моделей подобных экспериментов на компьютере. Статистические характеристики (медиана и стандартное отклонение) полученных оценок параметров были рассчитаны и сопоставлены для разных регрессионных моделей. Установлено, что модель нелинейной регрессии является более точной и надежной.

Ключевые слова: адсорбция, модель Ленгмюра, параметры, регрессия, компьютерное моделирование.

\section{Comparison of regression models for obtaining parameters of the adsorption isotherm of Langmuir's equation by method of modeling computer}

\author{
Nguen Phuc Cao, Vesentsev A.I., Dang Minh Thuy, Peristaja L.F. \\ Belgorod State National Research University, Belgorod
}

To obtain estimates of parameters of Langmuir adsorption isotherm from experimental data, researchers often use one of several linearized forms of the original non-linear equation to perform regression analysis. This technique has the advantage of calculation simplicity. However, by linearization, it ignores several fundamental prerequisite of the used least squares regression method, which can potentially lead to misleading results with higher level of uncertainties. In the last few decades, several suggestions for improvement have been made, one of which is using the non-linear regression technique, which can be done using merely Microsoft Excel. In this work, we performed a standard experiment of constructing the Langmuir adsorption isotherm of ions $\mathrm{Pb}^{2+}$ on montmorillonite clays from various regions (Polyanka, Nelidovka Belgorod, Russia and Lam Dong - Vietnam), then compared the parameter estimates, obtained using least squares method on two traditional linear forms and one non-linear form of the equation. After that, we constructed $10^{4}$ similar experiments on computer using Monte-Carlo simulation method, each one is performed on various levels of measurement errors, using identical data from the real experiment. Distribution curves and statistical characteristics (median and standard deviation) of the obtained parameter estimates have been calculated and compared for different regression models and different sorbents. The errors in estimating the parameters grow up almost linearly with the measurement errors for all regression models, and the non-linear model has the lowest slope coefficients for both parameters. It has been concluded that the non-linear regression model is far more accurate and reliable, therefore it is recommended using in research practice.

Keywords: adsorption, model Langmuir, parameters, regression, computer modelling. 


\section{Введение}

Изотерма Ленгмюра является одной из наиболее часто используемых моделей для описания явления адсорбции, благодаря своей простоте и солидной теоретической основе [1-2]. Первоначально предложенная Ирвингом Ленгмюром (1881-1957) почти сто лет назад для объяснения адсорбции молекул газа на однородной твердой поверхности, модель хорошо описывает разные другие процессы адсорбции с различными механизмами, в том числе адсорбции в растворах, несмотря на отклонения от первоначальных теоретических предположений (однородность поверхности адсорбента, однослойное покрытие, отсутствие взаимодействий между молекулами адсорбата и т.д.) [1-3]. Модель связывает количество адсорбированных молекул на единицу массы (или площади) адсорбента и равновесной концентрацией свободного адсорбата в объеме по следующему уравнению:

$$
A=\frac{A_{\infty} K C}{1+K C}
$$

Величина адсорбции А рассчитывается по формуле:

$$
A=\left(C_{o}-C\right) \cdot V_{m},
$$

где $\mathrm{A}$ - адсорбция; $A_{\infty}$ - предельная адсорбция; $\mathrm{K}$ - константа адсорбции Ленгмюра; $\mathrm{C}$ - равновесная концентрация; $\mathrm{C}_{\mathrm{o}}$ - исходная концентрация; $\mathrm{V}_{\mathrm{m}}$ - объем раствора, приведенный к массе сорбента. Величина $\mathrm{A}_{\infty}$ соответствует максимальной емкости монослоя, а К - константа адсорбционного равновесия.

Регрессионный анализ предназначен для определения параметров какой-либо теоретической модели из экспериментальных данных. Самым распространенным способом определения параметров уравнения регрессии является метод наименьших квадратов [4]. Сущность этого метода заключается в следующем: пусть имеются некоторая главная функция $\mathrm{y}=\mathrm{f}(\mathrm{x} ; \theta)$, где $\mathrm{x}-$ независимая (предикторная, пояснительная, входная) переменная; у - зависимая (отвечающая, объяснительная, выходная) переменная; $\theta$ - вектор параметров; и несколько точек данных $\left(\mathrm{x}_{\mathrm{i}}, \mathrm{y}_{\mathrm{i}}\right)$, полученных из эксперимента, которые отклоняются от истинных неизвестных точек по некоторым случайным ошибкам $\varepsilon_{\mathrm{i}}$. Метод наименьших квадратов позволяет определить статистически лучшие оценки параметров, если выполняются некоторые предположения [3-4]:

- значения независимой переменной $\mathrm{x}_{\mathrm{i}}$ известны точно, без ошибок;

- ошибки, связанные с зависимой переменной, имеют нулевые средние значения и подчиняются нормальному распределению;

- ошибки имеют постоянную дисперсию при разных $\mathrm{x}_{\mathrm{i}}$ (условие гомоскедастичности);

- погрешность одного измерения уі не влияет на ошибки других измерений, т.е. не коррелируют и не зависят от х.

При этих условиях, лучшие оценки параметров можно найти путем минимизации так называемой объективной функции - суммы квадратов ошибок (SSE - Sum of Squared Errors):

$$
S S E=\sum_{i} w_{i}\left(y_{i}-f\left(x_{i} ; \theta\right)\right)^{2},
$$

где $\mathrm{w}_{\mathrm{i}}$ - коэффициенты (весы) для устранения гетероскедастичности (неоднородность случайной погрешности между разными экспериментальными точками). Если $\mathrm{f}$ - линейная функция в виде $\mathrm{y}=\mathrm{mx}+\mathrm{b}$, то метод является линейным, для которого существует решение в явном виде. В общем, функция $\mathrm{f}$ может быть в любом виде (линейном или нелинейном), а решение находят численным алгоритмом. Численная

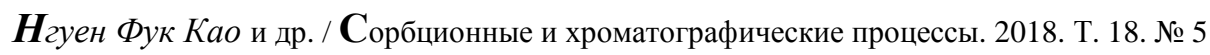


минимизация объективной функции может осуществляться с помощью надстройки «Поиск решения» программы Microsoft Excel [5].

Поскольку модель адсорбции Ленгмюра является нелинейной, обычно ее линеаризуют при обработке экспериментальных данных для получения параметров. По крайней мере, существуют четыре линеаризованные версии уравнения (1). Часто используют две из них, так называемые «двойная обратная» и «обратная по у» (табл. 1.). Как физические модели, все линеаризованные уравнения, вместе с уравнением (1), являются эквивалентными, описывающими одно и то же явление. Однако, с точки зрения статистики, как регрессионные модели, они отличаются. Это является причиной того, что использование разных версий уравнения Ленгмюра образует разные параметры из одного и того же набора экспериментальных данных [5-7]. Линеаризация моделей является чрезвычайно популярной практикой во всех отраслях науки. К сожалению, исследователи часто неправильно применяют метод, не уделяя адекватного внимания к лежащим в основе статистическим предположениям, что приводит к ошибочным, смещенным результатам [8, 9].

Модель адсорбции Ленгмюра имеет несколько статистических проблем, которые часто игнорируют $[5,7,10]$. Если используется линеаризация, она должна сопровождаться многократной повторной процедурой взвешивания для учета арифметического преобразования [11]. Проблемой, существующей для всех подходов, является то, что, будучи измеряемой величиной, равновесная концентрация адсорбата не известна без ошибок. Существует и другая проблема, а именно, равновесная концентрация не является истинной независимой переменной. Она дополнительно связана с адсорбцией по уравнению (2). В результате их ошибки автоматически коррелируют, что является нарушением условия применения метода наименьших квадратов. В своей работе, Босуэлл и Уокер [12] приняли другой подход: уравнение Ленгмюра (1) может быть объединено с (2) так, чтобы выражать адсорбцию как функцию от начальной концентрации - истинной независимой переменной (модель «нелинейная по $\mathrm{C}_{\mathrm{o}} \gg$ - см. табл. 1$)$.

Таблица 1. Регрессионные модели адсорбции Ленгмюра

\begin{tabular}{|c|c|}
\hline $\begin{array}{c}\text { Регрессионная } \\
\text { модель адсорбции }\end{array}$ & Форма уравнения \\
\hline Двойная обратная & $\frac{1}{A}=\frac{1}{A_{\infty}}+\frac{1}{K A_{\infty}} \cdot \frac{1}{C}$ \\
\hline Обратная по у & $\frac{C}{A}=\frac{C}{A_{\infty}}+\frac{1}{K A_{\infty}}$ \\
\hline Нелинейная по С & $A=\frac{1}{2}\left(A_{\infty}+\frac{V_{m}}{K}+V_{m} C_{o}-\sqrt{-4 A_{\infty} V_{m} C_{o}+\left(A_{\infty}+\frac{V_{m}}{K C}+V_{m} C_{o}\right)^{2}}\right)$ \\
\hline
\end{tabular}

Естественно возникает вопрос о сравнении выше приведенных регрессионных моделей адсорбции, ответ на который может быть получен с помощью метода компьютерного моделирования (или компьютерной симуляции) Монте-Карло. Метод широко используется в физике, статистике, финансах и бизнесе и других областях естественных наук для сравнения моделей, проверки гипотез и предсказания. Преимущества метода заключаются в возможности контролировать все условия эксперимента и способность образовать большое количество повторений в течение относительно короткого времени при низкой стоимости метода [13].

В данной работе попытаемся сравнивать методом компьютерного моделирования регрессионные модели получения параметров уравнения изотермы Ленгмюра 
с параметрами, полученными в аналогичных с реальными условиях эксперимента. Схема, приведенная на рис. 1, показывает соответствие реального и симуляционного экспериментов адсорбции.

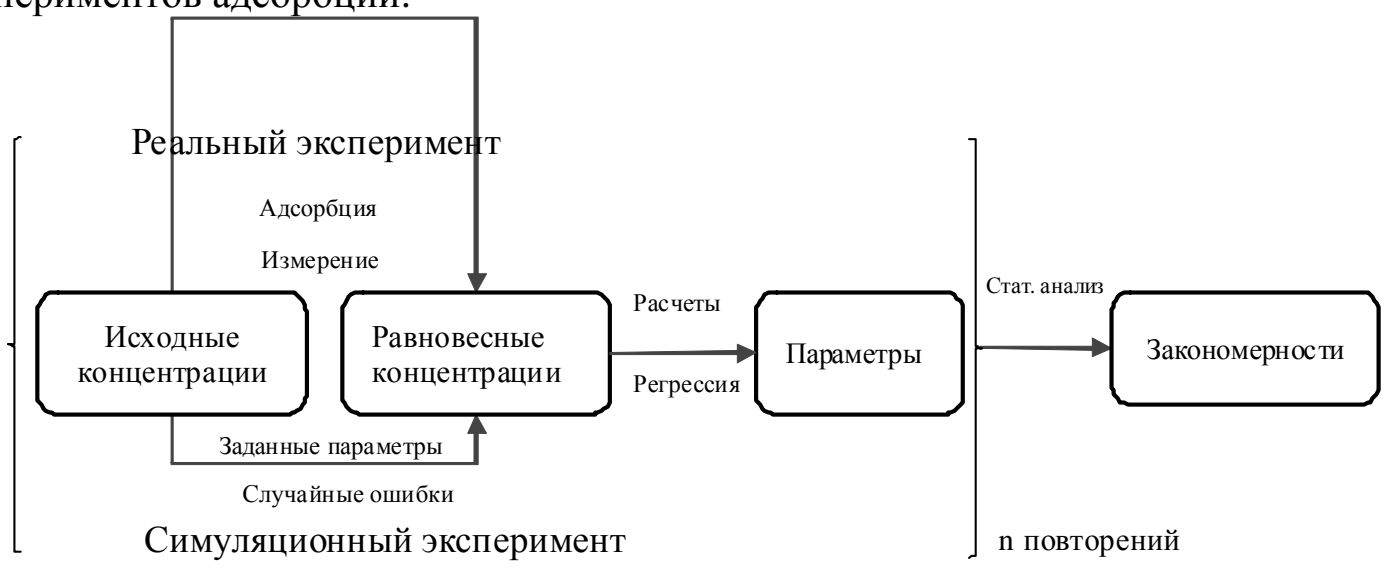

Рис. 1. Соответствие реального и симуляционного экспериментов адсорбции

Для получения равновесной концентрации, вместо реальных процессов адсорбции и измерения, компьютер вычисляет ее по заданным параметрам и добавляет к ней погрешность случайным образом.

\section{Эксперимент}

В качестве сорбентов использовали природные монтмориллонитовые глины месторождений Поляна и Нелидовка Белгородской области (Россия) и месторождения Там Бо провинции Лам Донг (Вьетнам). Природные глины предварительно высушили в сушильном шкафу при температуре $105 \pm 5^{\circ} \mathrm{C}$ до постоянной массы. Затем глины измельчали в диссольвере до порошкообразного состояния и просеивали через сито с диаметром ячеек 200 мкм.

В качестве сорбата использовали растворы ионов $\mathrm{Pb}^{2+}$, приготовленные из соли $\mathrm{Pb}\left(\mathrm{NO}_{3}\right)_{2}$ квалификации ч.д.а. Исходная концентрация ионов $\mathrm{Pb}^{2+}$ находилась в диапазоне 80-440 мкг/дм ${ }^{3}$. Равновесную концентрацию растворов ионов $\mathrm{Pb}^{2+}$ определяли на спектрофотометре Specord 50 с помощью реакции комплексообразования ионов $\mathrm{Pb}^{2+}$ с сульфарсазеном по ГОСТ 18293-72. Градуировочный график представлен на рис. 2.

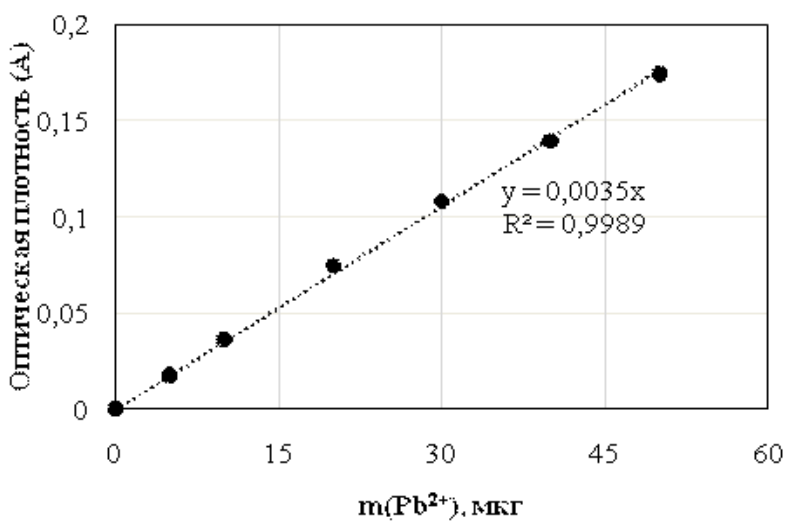

Рис. 2. Градуировочный график для количественного определения ионов свинца (II)

Оценочная погрешность определения содержания ионов $\mathrm{Pb}^{2+}$ соответствует случайным колебаниям оптической плотности при повторных измерениях и состав- 
ляет 0.5 мкг. Для получения погрешности к каждой равновесной концентрации, общая погрешность делится на соответствующий приведенный объем аликвоты в эксперименте.

В симуляционном эксперименте были взяты исходные параметры, строго соответствующие условиям реального эксперимента (исходные концентрации, объем раствора сорбата, масса сорбента, предельная адсорбция и константа адсорбционного равновесия). Проверяли эффективность регрессионных моделей в диапазоне погрешности эксперимента 0.1-0.9 мкг $\mathrm{Pb}^{2+}$. Симуляционные опыты проведены с помощью компьютерной программы, написанной нами на языке программирования Mathematica. Число симуляционных опытов равно 10000.

\section{Обсуждение результатов}

На рисунке 3 представлены изотермы адсорбции ионов $\mathrm{Pb}^{2+}$ на исследуемых сорбентах в разных системах координат.

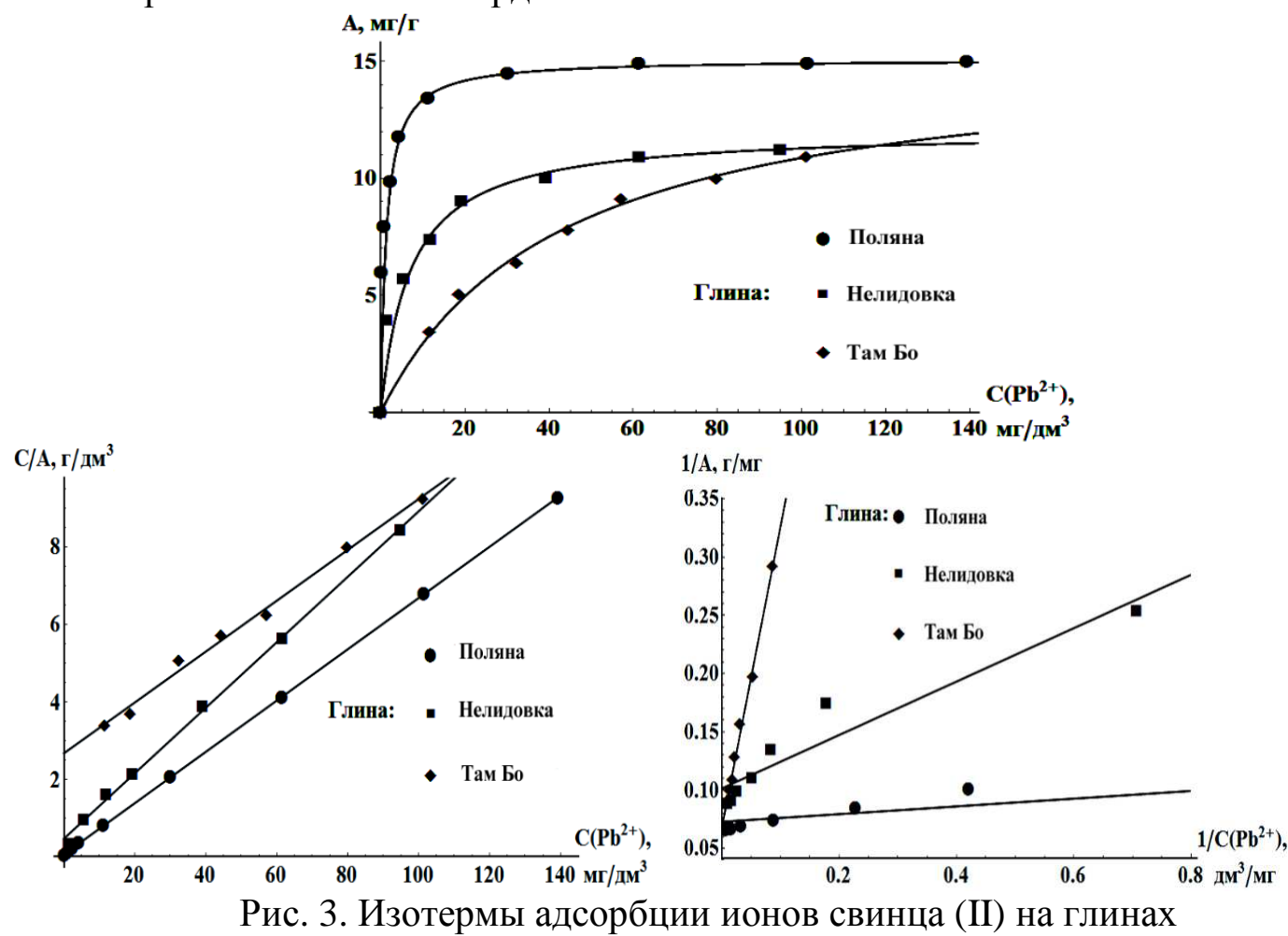

Из рис. 3 следует, что адсорбция ионов свинца (II) на всех исследованных адсорбентах соответствует модели мономолекулярной адсорбции Ленгмюра. Оценки параметров уравнения Ленгмюра, определенные разными регрессионными моделями, показаны в таблице 2 .

Для всех адсорбентов наблюдается тенденция уменьшения адсорбционной емкости, определенной регрессионными моделями по ряду «нелинейная по $\mathrm{C}_{0}$ », «обратная по у» и «двойная обратная», а константа сорбционного равновесия имеет тенденцию увеличиваться. Симуляционные опыты для Полянской глины были проведены с заданными параметрами уравнения Ленгмюра $\mathrm{A}_{\infty}=15.1$ мг/г и $\mathrm{K}=0.7692$ дм $^{3} / \mathrm{Mг}$. Кривые распределения параметров, полученных тремя регрессионными моделями, и их статистические показатели для Полянской глины при погрешности эксперимента 0.5 мкг $\mathrm{Pb}^{2+}$ представлены на рис. 4 и в табл. 3 . 
Таблица 2. Оценки параметров уравнения изотермы Ленгмюра

\begin{tabular}{|c|c|c|c|c|}
\hline \multirow{2}{*}{$\begin{array}{c}\text { Сорбент } \\
\text { месторождения }\end{array}$} & \multirow[b]{2}{*}{ Параметр } & \multicolumn{3}{|c|}{ Модель регрессии } \\
\hline & & Нелинейная по $\mathrm{C}_{\mathrm{o}}$ & Обратная по у & $\begin{array}{l}\text { Двойная } \\
\text { обратная }\end{array}$ \\
\hline \multirow{3}{*}{ Поляна } & $\mathrm{A}_{\infty}, \mathrm{M} \Gamma / \Gamma$ & 15.1 & 14.9 & 12.9 \\
\hline & $\mathrm{K}$, дм$^{3} / \mathrm{M \Gamma}$ & 0.77 & 1.10 & 2.50 \\
\hline & $\mathrm{R}^{2}$ & 0.9999 & 0.9999 & 0.9402 \\
\hline \multirow{3}{*}{ Нелидовка } & $\mathrm{A}_{\infty}, \mathrm{M \Gamma} / \Gamma$ & 12.0 & 11.9 & 9.9 \\
\hline & $\mathrm{K}, \mathrm{LM}^{3} / \mathrm{M \Gamma}$ & 0.14 & 0.18 & 0.44 \\
\hline & $\mathrm{R}^{2}$ & 0.9995 & 0.9982 & 0.9157 \\
\hline \multirow{3}{*}{$\begin{array}{l}\text { Там Бо, } \\
\text { Лам Донг }\end{array}$} & $\mathrm{A}_{\infty}, \mathrm{M \Gamma} / \Gamma$ & 15.4 & 15.3 & 14.8 \\
\hline & $\mathrm{K}$, дм$^{3} / \mathrm{мг}$ & 0.023 & 0.025 & 0.026 \\
\hline & $\mathrm{R}^{2}$ & 0.9998 & 0.9936 & 0.9940 \\
\hline
\end{tabular}
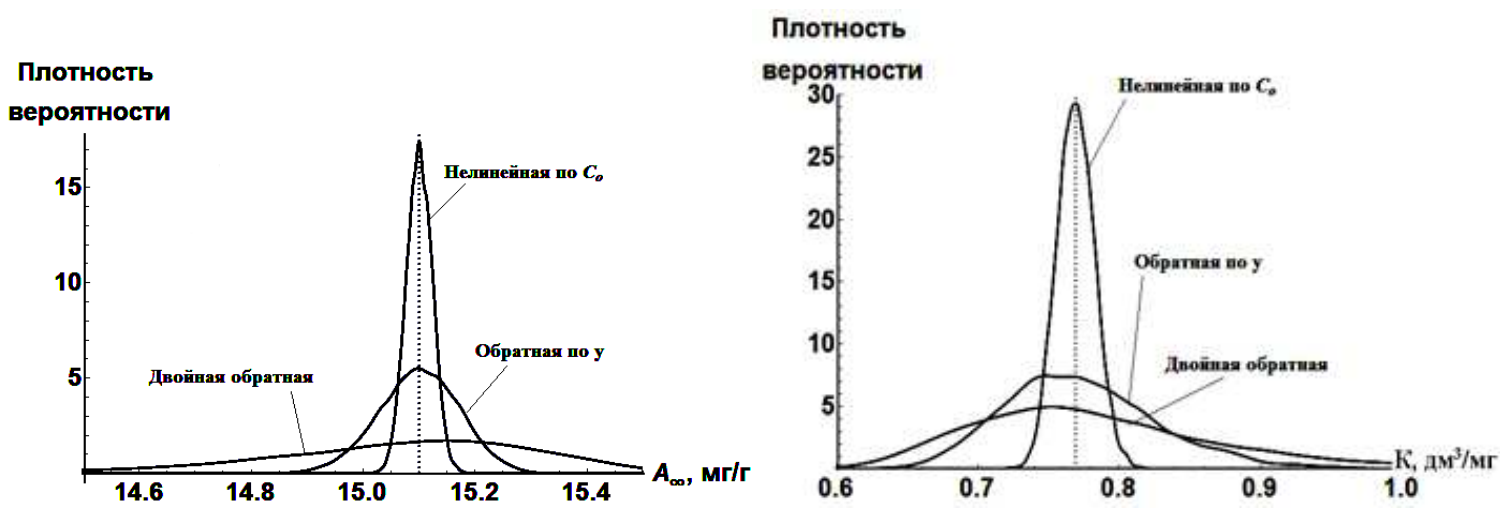

Рис. 4. Кривые распределения параметров уравнения изотермы Ленгмюра при погрешности эксперимента 0.5 мкг $\mathrm{Pb}^{2+}$

Таблица 3. Статистические характеристики распределений адсорбционных параметров

\begin{tabular}{|c|c|c|c|c|}
\hline \multirow[b]{2}{*}{ Показатель } & \multirow[b]{2}{*}{ Параметр } & \multicolumn{3}{|c|}{ Регрессионная модель } \\
\hline & & $\begin{array}{c}\text { Нелинейная } \\
\text { по } \mathrm{C}_{\mathrm{o}}\end{array}$ & Обратная по у & $\begin{array}{l}\text { Двойная } \\
\text { обратная }\end{array}$ \\
\hline \multirow{2}{*}{ Медиана } & $\mathrm{A}_{\infty}, \mathrm{M \Gamma} / \Gamma$ & 15.0996 & 15.0965 & 15.0955 \\
\hline & $\mathrm{K}$, дм $^{3} / \mathrm{M \Gamma}$ & 0.7693 & 0.7716 & 0.7723 \\
\hline \multirow{2}{*}{$\begin{array}{c}\text { Стандартное от- } \\
\text { клонение }\end{array}$} & $\mathrm{A}_{\infty}, \mathrm{M \Gamma} / \Gamma$ & 0.02336 & 0.07206 & 0.24402 \\
\hline & $\mathrm{K}$, дм$^{3} / \mathrm{M \Gamma}$ & 0.01332 & 0.05575 & 0.09433 \\
\hline \multirow{2}{*}{$\begin{array}{c}\text { Наименьшее } \\
\text { значение }\end{array}$} & $\mathrm{A}_{\infty}, \mathrm{M \Gamma} / \Gamma$ & 15.0009 & 14.7934 & 13.8650 \\
\hline & $\mathrm{K}$, дм $^{3} / \mathrm{M \Gamma}$ & 0.7161 & 0.6015 & 0.5542 \\
\hline \multirow{2}{*}{$\begin{array}{c}\text { Наибольшее зна- } \\
\text { чение }\end{array}$} & $\mathrm{A}_{\infty}, \mathrm{M \Gamma} / \Gamma$ & 15.2122 & 15.4281 & 15.7662 \\
\hline & $\mathrm{K}$, дм$^{3} / \mathrm{мг}$ & 0.8250 & 1.0899 & 1.4795 \\
\hline
\end{tabular}

В качестве показателя точности регрессионных моделей использовали медиану распределения. По сравнению с заданными параметрами, все модели показывают тенденцию переоценивать $\mathrm{A}_{\infty}$ и недооценивать $\mathrm{K}$, хотя смещенность параметров очень незначительна. Это может быть связано с ограниченным количеством экспериментальных точек для построения изотермы. При стремлении чисел экспериментальных точек к бесконечности можно ожидать, что оценки параметров будут более точными.

Ширине кривых распределения, а также достоверности регрессионных моделей отвечает стандартное отклонение. Чем больше стандартное отклонение, тем шире кривая распределения, тем менее достоверна модель. Анализируя рис. 4. и табл. 3, 
установили, что модель «нелинейная по $\mathrm{C}_{\mathrm{o}}$ » является самой достоверной, а модель «обратная по у» более достоверна, чем модель «двойная обратная». Это можно объяснить двумя причинами. Во-первых, использование нелинейной модели регрессии не нарушает (или нарушает в меньшей мере) статистические предпосылки метода наименьших квадратов. Во-вторых, после линеаризации часть экспериментальных точек часто находятся близко друг к другу (рис. 3), оказывая негативное влияние на достоверность определения параметров регрессионной прямой.

Зависимости достоверности оценки параметров разными регрессионными моделями от погрешности эксперимента для Полянской глины представлены на рис. 5. Для обоих параметров стандартное отклонение линейно увеличивается с увеличением погрешности эксперимента. Единственным исключением является зависимость стандартного отклонения константы адсорбционного равновесия, определенной моделью «двойной обратной», где она принимает вид параболы, показанной на указанном рисунке. Чем больше погрешность эксперимента, тем больше расходятся модели.
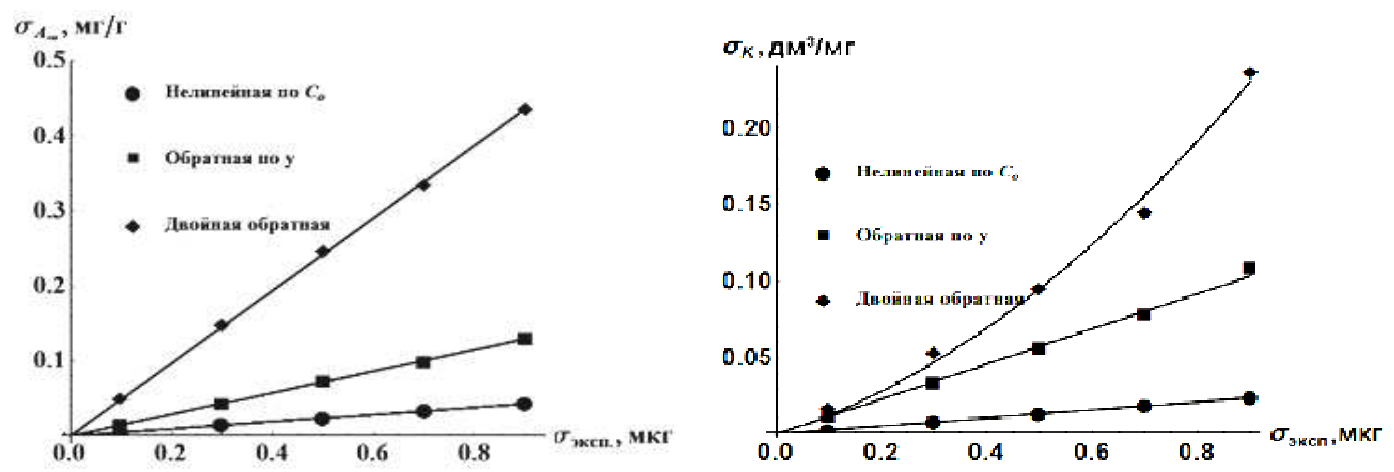

Рис. 5. Зависимость стандартного отклонения параметров от погрешности эксперимента

Для других адсорбентов с другими значениями адсорбционных параметров наблюдались аналогичные закономерности. Во всех случаях нелинейная модель обладает самой высокой достоверностью.

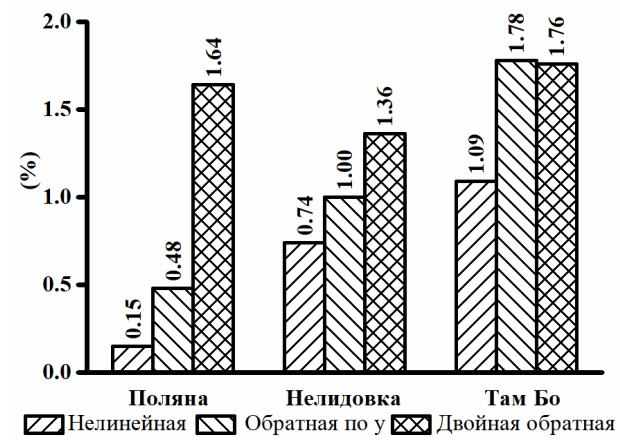

a

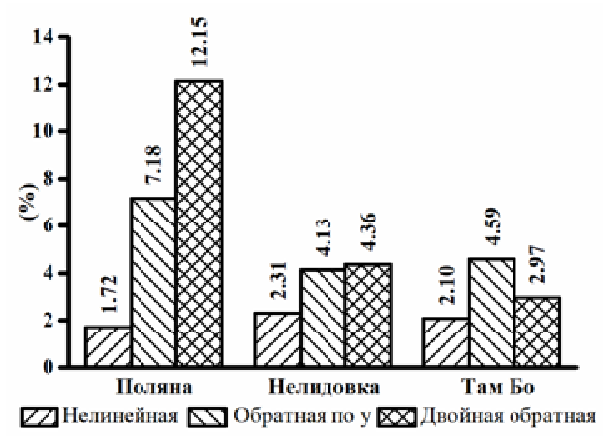

б

Рис. 6. Коэффициенты вариации параметров для исследуемых адсорбентов:

$$
\mathrm{a}-\mathrm{A}_{\infty} ; \sigma-\mathrm{K} \text {. }
$$

На рис. 6. представлены коэффициенты вариации (или относительная погрешность) параметров для разных адсорбентов. Коэффициент вариации есть отношение стандартного отклонения к среднему значению популяции и используется для сравнения распределений с разными средними значениями. Видно, что для всех адсорбентов нелинейная модель регрессии дает самые низкие коэффициенты обоих 
параметров. Отмечается случай для Полянской глины, где относительная погрешность оценки $\mathrm{A}_{\infty}$ меньше $0.2 \%$. Это связано с тем, что для данного адсорбента было определено большее количество экспериментальных точек в области высоких концентраций. Для других адсорбентов этот параметр оценивается с меньшей достоверностью.

\section{Заключение}

Таким образом, проведенными нами симуляционными экспериментами адсорбции ионов $\mathrm{Pb}^{2+}$ на трех адсорбентах в аналогичных с реальными условиях установлено, что регрессионная модель «нелинейная по $\mathrm{C}_{\mathrm{o}}$ » более достоверна, чем классические модели, и рекомендуется ее применение в исследованиях, основанных на использовании уравнения Ленгмюра.

\section{Список литературы/References}

1. Dabrowski A., Advances in Colloid and Interface Science, 2001, Vol. 93, pp. 135-224.

2. Sohn S., Kim D., Chemosphere, 2005, Vol. 58, pp. 115-123.

3. Kinniburgh D.G., Environ. Sci. Technoi., 1988, Vol. 20, pp. 895-904.

4. Wolberg J., Data Analysis Using the Method of Least Squares. Springer, 2006, p. 250.

5. El-Khaiary M.I., Journal of Hazardous Materials, 2008, Vol. 158, pp. 73-87.

6. Kemmer G., Keller S., Nature Protocols, 2010, Vol. 5, pp. 267-281.

Нгуен Фук Као - магистр Института инженерных технологий и естественных наук, ФГАОУ ВО «Белгородский государственный национальный исследовательский университет», НИУ «БелГУ», Белгород

Везенцев Александр Иванович - д.т.н., профессор, зав, кафедрой общей химии Института инженерных технологий и естественных наук, ФГАОУ ВО «Белгородский государственный национальный исследовательский университет», НИУ «БелГУ», Белгород

Данг Минь Тхуи - аспирант 3 года обучения, ФГАОУ ВО «Белгородский государственный национальный исследовательский университет», НИУ «БелГУ», Белгород

Перистая Лидия Федотовна - доцент кафедры общей химии Института инженерных технологий и естественных наук, ФГАОУ ВО «Белгородский государственный национальный исследовательский университет», НИУ «БелГУ», Белгород
7. Bolster C.H., Hornberger G.M., Soil Science Society of America journal, 2007, Vol. 71, pp. 1796-1806.

8. Otto Exner, J. Phys. Org. Chem., 1997,

Vol. 10, pp. 797-813.

9. Zielinski T.J, Allendoerder R.D., J. Chem.

Educ., 1997, Vol. 74, pp. 1001-1007.

10. Tellinghuisen J., Methods in Enzymology, 2009, Vol. 467, pp. 499-529.

11.Lemcoff N.O., The Chemical Engineering Journal, 1977, Vol. 13, pp. 71-74.

12.Bothwell M.K., Walker L.P., Bioresource Technology, 1995, Vol. 53, pp. 21-29.

13. Harrison R.L., AIP conference proceedings, 2010, Vol. 1204, pp. 17-21.

Nguen Phuc Cao - magister, Federal State Autonomous Educational Institution of Higher Education «Belgorod National Research University», Belgorod

Vezentsev Aleksandr I. - doctor of technical sciences, professor, head of department of the general chemistry of Institute of engineering technologies and natural sciences, Federal State Autonomous Educational Institution of Higher Education «Belgorod National Research University», Belgorod, E-mail: vesentsev@bsu.edu.ru

Dang Minh Thuy - PhD student of the $3^{\text {sd }}$ year, Federal State Autonomous Educational Institution of Higher Education «Belgorod National Research University», Belgorod, E-mail: thuybsu@gmail.com

Peristaya Lidiya F. - assistant professor of of department of the general chemistry of Institute of engineering technologies and natural sciences, Federal State Autonomous Educational Institution of Higher Education «Belgorod National Research University», Belgorod, E-mail: peristaya@bsu.edu.ru 